\section{Hipovitaminosis D en población portadora de enfermedad inflamatoria intestinal del Sur de Chile}

\author{
GLORIA HORTA ${ }^{1}$, SEBASTIÁN SOTO ${ }^{2}$, GONZALO LABARCA $^{2}$
}

\section{Vitamin D levels in patients with inflammatory bowel disease}

Background: Inflammatory Bowell Diseases (IBD), either ulcerative colitis (UC) or Chron's disease (CD) are commonly associated with intestinal malabsorption, and insufficient levels 1-25 hydroxycholecalciferol (vitamin D). These insufficient levels could be linked with an increased risk of clinical disease activity. Aim: To report vitamin D levels in patients with IBD living in Southern Chile and their possible association with clinical disease activity. Material and Methods: A prospective, observational study in outpatients with IBD living in Los Angeles, Chile. Demographic data and clinical activity using clinical scores were recorded. Vitamin D levels and fecal calprotectin were measured. Results: We studied 44 patients, 32 (73\%) with UC and $12(27 \%)$ with CD. The median age at diagnosis was 33 years old. Forty-one (93\%) had mild-inactive disease and $3(7 \%)$ a moderate disease. Mean vitamin D levels were $15.2 \pm 7.1$ $\mathrm{ng} / \mathrm{ml}$. Twenty five percent of patients had sufficient levels (> $20 \mathrm{ng} / \mathrm{ml}$ ), 35\% insufficient levels and $40 \%$ poor levels. No association between these levels and disease activity was found. Conclusions: These patients with IBD had a high frequency of hypovitaminosis $D$.

(Rev Med Chile 2021; 149: 393-398)

Key words: Inflammatory Bowel Diseases; Vitamin D; Vitamin D Deficiency.
${ }^{1}$ Gastroenterología adulto. Complejo Asistencial Dr. Víctor Ríos Ruiz. Los Ángeles, Chile.

${ }^{2}$ Facultad de Medicina,

Universidad de Concepción. Los Ángeles, Chile.

Los autores declaran no tener conflicto de interés.

Trabajo no recibió financiamiento.

Recibido el 1 de septiembre de 2019, aceptado el 18 de marzo de 2021.

\section{Correspondencia a:} Dra. Gloria Horta Hermosilla, Vía del Río 3825 departamento 403, Chiguayante, Concepción. glhorta@gmail.com
I as enfermedades inflamatorias intestinales (EII) son enfermedades crónicas con alto impacto en morbilidad y nutrición ${ }^{1}$. La 1-25-hidroxicolecalciferol (vitamina D) participa activamente en la mineralización ósea y presenta además un rol como hormona "pleiotrópica". $\mathrm{Su}$ receptor se encuentra en todas las células nucleadas del organismo ${ }^{2}$ y se ha postulado que puede ejercer una acción inmunomoduladora aumentando la producción de citoquinas anti-inflamatorias que promueven la tolerancia inmune y disminuyen la inflamación ${ }^{3}$.

La vitamina $\mathrm{D}$ se encuentra en los alimentos y se produce en la dermis tras exposición a los rayos ultravioleta (UV). Una menor exposición UV resulta en niveles más bajos ${ }^{4}$, tal como ocurre en poblaciones alejadas geográficamente de la línea del Ecuador ${ }^{5,6}$.

Estudios postulan que hasta $60 \%$ de los pacientes con EII tendrían niveles bajos de vitamina D, pero su relación con la actividad y evolución de la enfermedad no está claro ${ }^{7,8}$.

Los objetivos del presente estudio son caracterizar a pacientes portadores de EII en el sur de Chile, conocer sus niveles de Vitamina D y la relación de ésta con su enfermedad.

\section{Materiales y Método}

Estudio observacional, prospectivo entre los años 2017-2018 en el Complejo Asistencial Dr. Víctor Ríos Ruiz, comuna de Los Ángeles, región 
de Bío-Bío. Se incluyeron pacientes mayores de 15 años en control ambulatorio por EII del tipo Colitis Ulcerosa (CU) y enfermedad de Crohn (EC). El diagnóstico debía estar establecido en base a clínica, laboratorio, colonoscopia e histología compatibles 9 . Se contó con la aprobación del Comité de Etica de la institución (Servicio de Salud Bío-Bío) y los pacientes interesados en participar firmaron el consentimiento informado correspondiente.

\section{Registro de variables}

Se registraron datos demográficos, autoreporte de pueblo originario de la zona (pehuenche) mediante encuesta, síntomas, exámen físico y exámenes de laboratorio. Se registraron variables: tipo de EII, extensión o fenotipo, años de evolución, manifestaciones extraintestinales, cirugías previas, número de hospitalizaciones por crisis y tratamiento actual. Se solicitaron exámenes: hemograma, velocidad de eritrosedimentación (VHS), proteína $\mathrm{C}$ reactiva (PCR), albúmina plasmática, niveles plasmáticos de vitamina $\mathrm{D}$ (VIDAS $^{\mathrm{R}} 25 \mathrm{OH}$, Vitamina D total, Biomerieux) y niveles cuantitativos de calprotectina fecal (CF) (RIDASCREEN ${ }^{\oplus}$ Calprotectin, R-Biopharm AG).

Los niveles de vitamina $\mathrm{D}$ se clasificaron de acuerdo con los estándares internacionales actuales definiendo las categorías: 1) niveles suficientes (> $20 \mathrm{ng} / \mathrm{ml}) ; 2$ ) niveles insuficientes (20-12 ng/ $\mathrm{ml})$ y 3$)$ niveles deficientes $(<12 \mathrm{ng} / \mathrm{ml})^{10}$.

La CF se consideró valores bajo $50 \mathrm{ug} / \mathrm{g}$ como sugerentes de enfermedad inactiva o en remisión ${ }^{11,12}$, valores sobre $600 \mathrm{ug} / \mathrm{g}$ como sugerente de enfermedad activa severa y valores intermedios como enfermedad activa, leve a moderada, ya existe variabilidad de interpretaciones en estos rangos descrita en diversas publicaciones ${ }^{11-15}$. Dado que el valor máximo entregado por RIDASCREEN ${ }^{\mathrm{R}}$ Calprotectin, R-Biopharm AG es de $600 \mathrm{ug} / \mathrm{g}$, los valores informados como $\geq 600 \mathrm{ug} / \mathrm{g}$ fueron considerados como valor absoluto $600 \mathrm{ug} / \mathrm{gr}$ para efectos de análisis estadístico.

\section{Desenlaces}

Como desenlace principal se definió caracterizar clinicamente a los pacientes, incluyendo el grado de severidad de la enfermedad, sus niveles plasmáticos de vitamina D y de CF. Para evaluar el grado de actividad de la enfermedad se clasificó cada participante como 1) inactivo; 2) actividad leve, 3) actividad moderada, y 4) actividad severa. El grado de actividad en pacientes con CU fue evaluado con el índice de severidad de Truelove-Witts y de Truelove-Witts modificado o Lichtiger ${ }^{16,17}$. Para pacientes portadores de EC se utilizó el Crohn's Disease Activity Index (CDAI $)^{18}$.

Como desenlaces secundarios se definió: correlacionar el grado de actividad de la enfermedad con los niveles de vitamina D y evaluar la concordancia entre los niveles de CF y vitamina D.

\section{Análisis estadístico}

Las variables continuas fueron expresadas como media y desviación estándar ( $\pm \mathrm{DS})$, mientras que las variables categóricas fueron expresadas como porcentaje. La media de las concentraciones de vitamina $\mathrm{D}$ de los pacientes con enfermedad activa e inactiva fueron analizados mediante prueba no paramétrica de U-Mann Whitney. Mientras que las variables categóricas fueron analizadas mediante test exacto de fisher. Además, se realizó un análisis de regresión lineal con índice de correlación $\mathrm{R}^{2}$ entre los valores de los niveles plasmáticos de vitamina $\mathrm{D}$ y los puntajes obtenidos mediante los scores de Truelove-Witts modificado, CDAI y los valores cuantitativos de CF.

Todos los análisis fueron realizados con el software IBM SPSS, version 22.0. (IBM Corp, $\mathrm{NY}, \mathrm{EEUU}$ ) y se definió como valor $\mathrm{p}$ significativo $<0,05$.

\section{Resultados}

\section{Características demográficas}

Se registró un total de 44 pacientes, 2 pacientes perdieron seguimiento sobre niveles de vitamina $D$, y 6 respecto a niveles de calprotectina fecal. El reclutamiento y toma de muestras se obtuvieron en distintas épocas del año. El registro clínico se obtuvo dentro de los 7 días con respecto a las tomas de la muestra (sangre y deposición). Se encontró 31/44 (70\%) mujeres. Hubo 32/44 (73\%) pacientes con diagnóstico de CU y 12/44 (27\%) con EC, ninguno reportó ascendencia y/o pertenencia a comunidades de pueblo originario. La mediana de edad para el debut de la enfermedad fue de 33 años. El promedio de años de evolución fue de 9 años para CU y 5 años en EC. Al momento del análisis, 22/44 (50\%) reportaba haber tenido una crisis grave alguna vez, siendo 2 
veces más frecuente en EC que en CU. Respecto al fenotipo, en CU, el 54\% presentaba colitis extensa mientras que en el caso de la EC, el compromiso más frecuente fue ileocolonico con $67 \%$. Respecto al tratamiento, todos los pacientes portadores de CU estaban con aminosalicilatos (5-ASA) oral y/o rectal y $13 / 32(40 \%)$ con inmunosupresor (tiopurinas) en dosis media de $1,5 \mathrm{mg} / \mathrm{Kg} /$ día. Todos los pacientes con EC se encontraban con inmunosupresores (tiopurinas) en dosis media de 2,0 mg/Kg/día. Del total de la muestra, dos pacientes estaban con terapia corticoesteroidal y 4 pacientes con terapia biológica (infliximab ${ }^{\circledR}$ ) (Tabla 1).

\section{Actividad clínica, niveles de vitamina $D$ y CF}

El (93\%) de la muestra presentaba enfermedad leve o inactiva, mientras que 3 pacientes $(7 \%)$ se encontraban con actividad moderada y ningún paciente con actividad severa. En 40/44 (90,9\%) pacientes se obtuvo niveles de vitamina $\mathrm{D}$ cuyo resultado promedio fue de $15,2(\mathrm{DS} \pm 7,1) \mathrm{ng} / \mathrm{ml}$. 10 pacientes $(25 \%)$ presentaron niveles suficientes (> $20 \mathrm{ng} / \mathrm{ml}), 14 / 40$ (35\%) niveles insuficientes y
16/40 (40\%) niveles deficientes. Al agrupar ambos puntos de corte, $(75 \%)$ no tenía niveles adecuados o suficientes de vitamina $\mathrm{D}$.

No fue posible establecer correlación entre grado de actividad de la enfermedad y niveles de Vitamina D dado lo pequeño del grupo muestral y mayoritariamente con enfermedad inactiva o leve. $\mathrm{Al}$ analizar la muestra según tipo de EII, (CU y EC), aplicando sus scores de actividad respectivos (Truelove-Witts modificado y CDAI) tampoco se encontraron diferencias significativas entre valores de vitamina $\mathrm{D}$ y scores de actividad de la enfermedad (Figura 1 a y b).

No hubo diferencias significativas entre los valores de vitamina $\mathrm{D}$ obtenidos en invierno y los obtenidos en verano.

Finalmente, la cuantificación de CF se realizó en $38 / 44(86,3 \%)$ pacientes de los cuales 10 presentaron niveles $<50 \mathrm{ug} / \mathrm{g}$, todos concordantes con actividad clínica leve/inactiva, mientras que los 3 pacientes con actividad clínica moderada presentaron niveles $\geq 600 \mathrm{ug} / \mathrm{g}$. No encontramos relación entre valores de calprotectina fecal y valores de vitamina D (Figura $1 \mathrm{c}$ ).

Tabla 1. Características clínicas y demográficas de los pacientes incluidos en el análisis

\begin{tabular}{|c|c|c|c|}
\hline & $\begin{array}{c}\text { CU } \\
n=32(\%)\end{array}$ & $\begin{array}{c}E C \\
n=12(\%)\end{array}$ & $\begin{array}{c}\text { Total } \\
n=44\end{array}$ \\
\hline Edad, (DS \pm$)$ & $43(D S \pm 14)$ & 39 (DS \pm 18$)$ & 41 (10-80 años) \\
\hline Mujeres & $25(78 \%)$ & $6(50 \%)$ & \\
\hline Duración enfermedad en años & 9 (0-30 años) & 5 (0-12 años) & \\
\hline Hospitalizaciones por crisis & $12(37 \%)$ & $10(83 \%)$ & \\
\hline \multicolumn{4}{|l|}{ Síntomas extra intestinales } \\
\hline Articular & $10(31 \%)$ & $5(41 \%)$ & 15 \\
\hline Dermatológico & $2(6 \%)$ & $1(8 \%)$ & 3 \\
\hline Agentes biológicos (infliximab) & $0(0 \%)$ & $4(33 \%)$ & 4 \\
\hline 5 ASA local & $16(50 \%)$ & $1(8 \%)$ & 17 \\
\hline 5 ASA oral & $30(94 \%)$ & $6(50 \%)$ & 36 \\
\hline Corticoides & $2(6 \%)$ & $0(0 \%)$ & 2 \\
\hline Inmunosupresores & $13(40 \%)$ & $12(100 \%)$ & 25 \\
\hline Cirugía & $1(3 \%)$ & $4(33 \%)$ & 5 \\
\hline Vitamina D ng/ml & $15,7( \pm 7,3)^{*}$ & $13,7( \pm 6,9)^{\$}$ & 40 \\
\hline Niveles calprotectina fecal ug/g & $261,7^{x}$ & $135,4^{\$}$ & 38 \\
\hline
\end{tabular}

Abreviaciones: CU: colitis ulcerosa; EC: Enfermedad de Crohn; 5 ASA: 5- amino salicilatos, DS: Desviación estándar. *Sobre un total de 29 pacientes disponibles; \$: Sobre un total de 11 pacientes disponibles, X: Sobre un total de 27 pacientes disponibles. 
a)

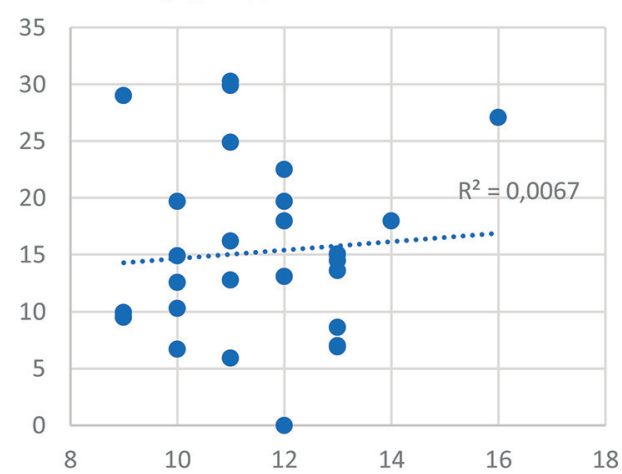

b)

Vit D (ng/ml)/CDAl score

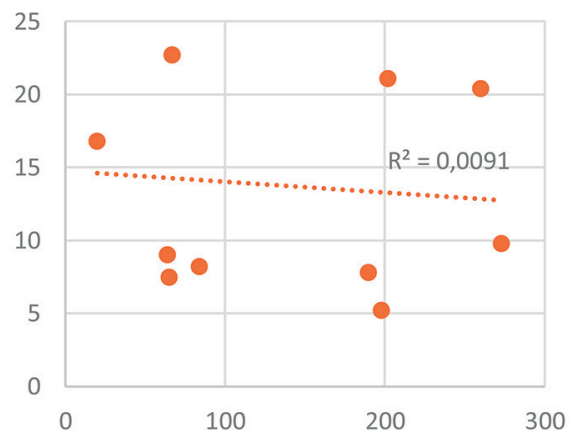

c)

Calprotectina fecal/Vit D

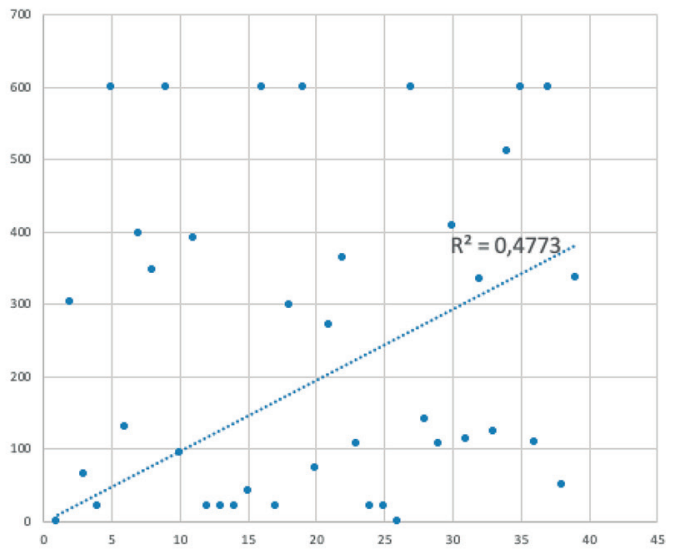

Figura 1. Gráfico de dispersión simple con ajuste de línea de niveles de vitamina $D$ por scores de actividad de enfermedad a) Truelove-Witts modificado score para Colitis Ulcerosa $\left(R^{2}=0,0067\right)$, b) CDAl score para Enfermedad de Crohn $\left(R^{2}=0,0091\right)$. (no significativo).

\section{Discusión}

Nuestro estudio caracterizó una población de pacientes portadores de EII en el sur de Chile con resultados similares a otros estudios de nuestro país ${ }^{19-21}$. No encontramos ascendencia de pueblos originarios, pese a encontrarnos en una de las regiones con mayor concentración de comunidades indígenas ${ }^{22}$.

La mayoría no tenía niveles adecuados de vitamina D. Esto está ampliamente descrito en ésta patología $7,8,23$. Una amplia revisión sistemática y metaanálisis concluyó que ser portador de una EII se asociaba con $64 \%$ más de probabilidades de tener déficit de vitamina $\mathrm{D}$ con respecto a la población general ${ }^{24,25}$.

Otro factor a considerar en nuestro grupo sería la ubicación geográfica. Nos ubicamos en el paralelo 37o respecto de la línea del Ecuador y diversos estudios han mostrado que a mayor latitud existe más riesgo de presentar niveles de vitamina D bajos ${ }^{26,27}$. Un registro de bases de datos nacional encontró una mayor tasa de hospitalizaciones por EII (CU o EC) a mayor latitud ${ }^{28}$.

Las principales limitaciones de este estudio son: El tamaño muestral pequeño, conformado por población ambulatoria y sin presencia de actividad severa. 
Como proyecciones, aún no sabemos si la deficiencia de vitamina $\mathrm{D}$ sería consecuencia de una mala absorción debido al daño de la mucosa y si acaso contribuiría a perpetuar éste daño y por lo tanto asociarse a una peor evolución y/o gravedad de la enfermedad. Tampoco sabemos cuáles serían los niveles más adecuados para estos pacientes ya que las recomendaciones actuales tienen relación con el metabolismo óseo, pero no con las funciones inmunomoduladoras ni, específicamente, para pacientes portadores de EII.

\section{Conclusión}

La hipovitaminosis D es un hallazgo frecuente en población portadora de EII en el sur de Chile, aún con actividad de enfermedad leve. Esos datos deberán reevaluarse en actividad severa y tras el efecto de la suplementación con vitamina $\mathrm{D}$.

\section{Referencias}

1. Baumgart DC, Sandborn WJ. Inflammatory bowel disease: clinical aspects and established and evolving therapies. Lancet. 2007; 369 (9573): 1641-57.

2. DeLuca HF. Overview of general physiologic features and functions of vitamin D. Am J Clin Nutr. 2004; 80 (6 Suppl): 1689S-96S.

3. Cantorna MT, Zhu Y, Froicu M, Wittke A. Vitamin D status, 1,25-dihydroxyvitamin D3, and the immune system. Am J Clin Nutr. 2004; 80 (6 Suppl): 1717S-20S.

4. Holick MF, MacLaughlin JA, Clark MB, Holick SA, Potts JT, Jr. Anderson RR, et al. Photosynthesis of previtamin D3 in human skin and the physiologic consequences. Science. 1980; 210 (4466): 203-5.

5. Cashman KD, Dowling KG, Skrabakova Z, Gonzalez-Gross M, Valtuena J, De Henauw S, et al. Vitamin D deficiency in Europe: pandemic? Am J Clin Nutr. 2016; 103 (4): 1033-44.

6. Macdonald HM, Mavroeidi A, Fraser WD, Darling AL, Black AJ, Aucott L, et al. Sunlight and dietary contributions to the seasonal vitamin D status of cohorts of healthy postmenopausal women living at northerly latitudes: a major cause for concern? Osteoporos Int. 2011; 22 (9): 2461-72.

7. Abraham BP, Prasad P, Malaty HM. Vitamin D deficiency and corticosteroid use are risk factors for low bone mineral density in inflammatory bowel disease patients. Dig Dis Sci. 2014; 59 (8): 1878-84.
8. Driscoll RH, Jr. Meredith SC, Sitrin M, Rosenberg IH. Vitamin D deficiency and bone disease in patients with Crohn's disease. Gastroenterology. 1982; 83 (6): 1252-8.

9. Matsuoka K, Kobayashi T, Ueno F, Matsui T, Hirai F, Inoue $\mathrm{N}$, et al. Evidence-based clinical practice guidelines for inflammatory bowel disease. J Gastroenterol. 2018; 53 (3): 305-53.

10. Giustina A, Adler RA, Binkley N, Bouillon R, Ebeling $\mathrm{PR}$, Lazaretti-Castro M, et al. Controversies in Vitamin D: Summary Statement From an International Conference. J Clin Endocrinol Metab. 2019; 104 (2): 234-40.

11. https://clinical.r-biopharm.com/wp-content/ uploads/sites/3/2012/06/g09036ridascreen-calprotectin2017-03-30_gb.pdf. Biopharm 2017 [Visitado el 2 de septiembre de 2020].

12. Waugh N, Cummins E, Royle P, Kandala NB, Shyangdan D, Arasaradnam R, et al. Faecal calprotectin testing for differentiating amongst inflammatory and non-inflammatory bowel diseases: systematic review and economic evaluation. Health Technol Assess. 2013; 17 (55): xv-xix, 1-211.

13. Poullis A, Foster R, Mendall MA, Fagerhol MK. Emerging role of calprotectin in gastroenterology. J Gastroenterol Hepatol. 2003; 18 (7): 756-62.

14. Bjarnason I. The Use of Fecal Calprotectin in Inflammatory Bowel Disease. Gastroenterol Hepatol (NY). 2017; 13 (1): 53-6.

15. Maaser C, Sturm A, Vavricka SR, Kucharzik T, Fiorino G, Annese V, et al. ECCO-ESGAR Guideline for Diagnostic Assessment in IBD Part 1: Initial diagnosis, monitoring of known IBD, detection of complications. J Crohns Colitis. 2019; 13 (2): 144-64.

16. Truelove SC, Witts LJ. Cortisone in ulcerative colitis; final report on a therapeutic trial. Br Med J. 1955; 2 (4947): 1041-8.

17. Lichtiger S, Present DH. Preliminary report: cyclosporin in treatment of severe active ulcerative colitis. Lancet. 1990; 336 (8706): 16-9.

18. Best WR, Becktel JM, Singleton JW, Kern F, Jr. Development of a Crohn's disease activity index. National Cooperative Crohn's Disease Study. Gastroenterology. 1976; 70 (3): 439-44.

19. Bellolio Roth F, Gómez J, Cerda J. Increase in Hospital Discharges for Inflammatory Bowel Diseases in Chile Between 2001 and 2012. Dig Dis Sci. 2017; 62 (9): 2311 7.

20. Figueroa CC, Quera PR, Valenzuela EJ, Jensen BC. [Inflammatory bowel disease: experience of two Chilean centers]. Rev Med Chil. 2005; 133 (11): 1295-304.

21. Simian D, Estay C, Lubascher J, Acuna R, Kronberg U, Figueroa C, et al. [Inflammatory bowel disease. Expe- 
rience in 316 patients]. Rev Med Chil. 2014; 142 (8): 1006-13.

22. Conadi. www.conadi.gob.cl 2019 [Available from: www. conadi.gob.cl. [Visitado el 2 de septiembre de 2020].

23. Siffledeen JS, Siminoski K, Steinhart H, Greenberg G, Fedorak RN. The frequency of vitamin D deficiency in adults with Crohn's disease. Can J Gastroenterol. 2003; 17 (8): 473-8.

24. Del Pinto R, Pietropaoli D, Chandar AK, Ferri C, Cominelli F. Association Between Inflammatory Bowel Disease and Vitamin D Deficiency: A Systematic Review and Meta-analysis. Inflamm Bowel Dis. 2015; 21 (11): 2708-17.

25. Ulitsky A, Ananthakrishnan AN, Naik A, Skaros S, Zadvornova Y, Binion DG, et al. Vitamin D deficiency in patients with inflammatory bowel disease: association with disease activity and quality of life. JPEN J Parenter Enteral Nutr. 2011; 35 (3): 308-16.

26. Leary PF, Zamfirova I, Au J, McCracken WH. Effect of Latitude on Vitamin D Levels. J Am Osteopath Assoc. 2017; 117 (7): 433-9.

27. Genuis SJ, Schwalfenberg GK, Hiltz MN, Vaselenak SA. Vitamin D status of clinical practice populations at higher latitudes: analysis and applications. Int J Environ Res Public Health. 2009; 6 (1): 151-73.

28. Jaime F, Riutort MC, Alvarez-Lobos M, Hoyos-Bachiloglu R, Camargo CA, Jr. Borzutzky A. Solar radiation is inversely associated with inflammatory bowel disease admissions. Scand J Gastroenterol. 2017; 52 (6-7): 7307.

\section{ARTE Y FOTOGRAFÍA}

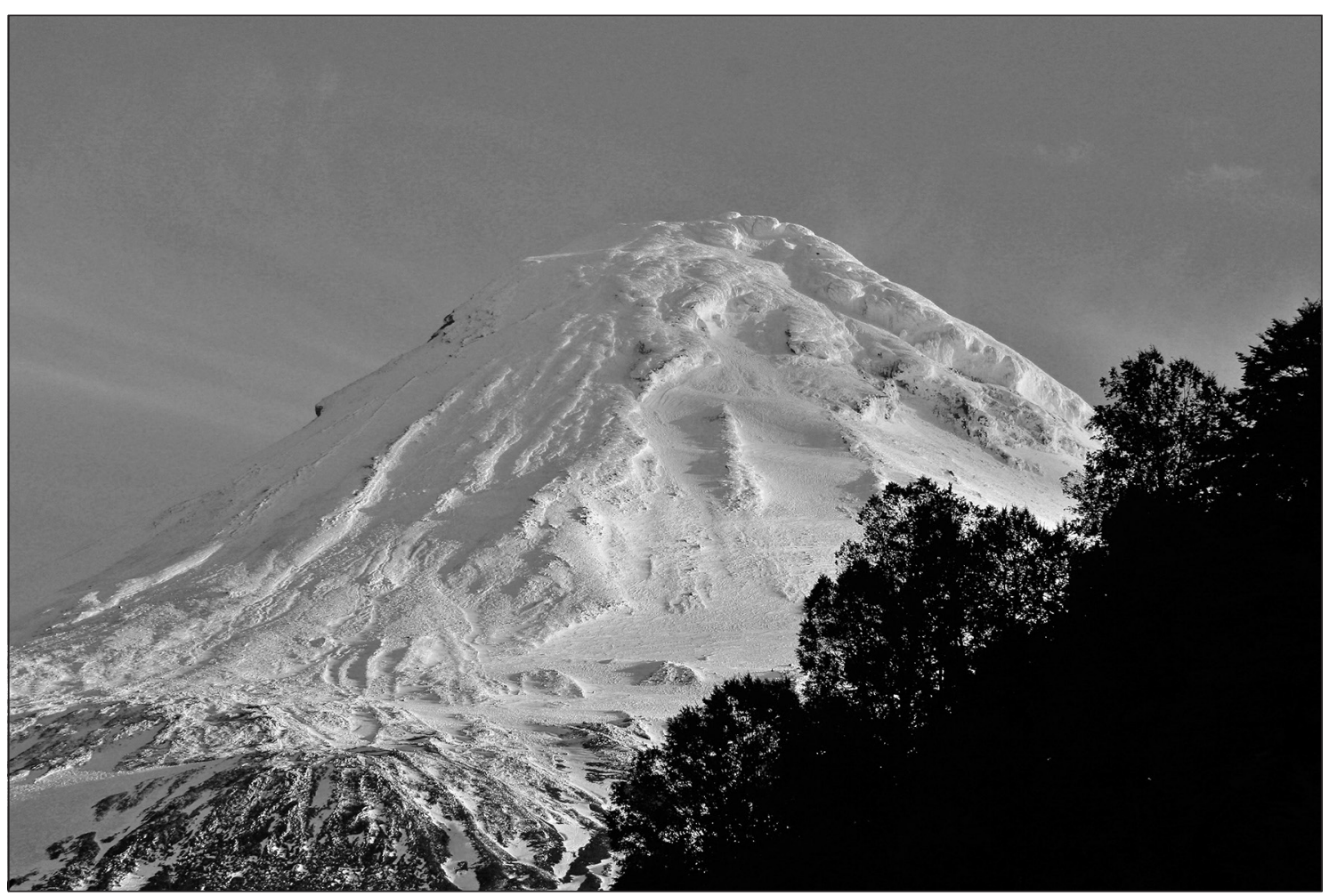

Sendero Momolluco, Curarrehue, La Araucanía. Dr. Jorge Sapunar Zenteno 